\title{
SLIDING MODE CONTROL OF LINEAR SYNCHRONOUS MOTOR SERVODRIVE
}

\author{
Abdel-Magid M. A. Mohamed \\ Electrical Engineering Department, Aswan Faculty of Engineering, \\ South Valley University, Aswan, Egypt
}

(Received April 12, 2006. Accepted May 13, 2006)

\begin{abstract}
This paper proposes a method to design sliding mode control (SMC) for the position tracking control of a permanent magnet linear synchronous motor (PMLSM). The controller is designed to achieve accurate performance in the presence of unknown disturbance and parameter uncertainties. The components of the control law, the equivalent component and the robust component, are designed such that the nominal system exhibits desirable dynamics and the reaching condition is guaranteed. Switching surface is designed based on pole placement and generalized matrix inverse. Simulation results show that the suggested procedure provides high performance dynamic characteristics and robust with regard to external disturbance and parameter variations.
\end{abstract}

KEYWORDS: Sliding mode control, Pole assignment, Descriptor systems, Control of electric drives, Observers,

\section{INTRODUCTION}

The control of motors used in high performance servo drives is one of the most fundamental problems that considered in the field of automatic control. The controller design for a plant must satisfy that the outputs are tracking any input reference value and must be independent of the parameter uncertainty and disturbances as much as possible.

Variable structure control with sliding mode has been known for its robustness properties such as insensitivity to parameter variations, external disturbance rejection and satisfactory dynamic responses [see, e.g. 1-3]. These robustness properties make sliding mode control a good candidate for industrial applications. Sliding mode control generally involves two main steps: firstly, the selection of a sliding surface which induces assigned stable reduced order dynamics, and secondly, the synthesis of a control law to force the closed loop system trajectories onto and subsequently remain on the sliding surface [4-6]. Several researches deal with the design and the applications of SMC on the control of motors [e.g. 1,3,7,8]. In [1] a proposed sliding mode control method based motion control drive design in accordance with a trapezoidal velocity profile for permanent magnet synchronous motor is given. A robust controller is designed in [7] by using proportional integral (PI) control algorithm 
and a sliding mode control. In [8] a robust controller is designed by employing variable structure and linear quadratic method for PMSM. Also, robust control method for PMLSM based on recurrent fuzzy neural network using sliding mode control is given in [3].

In this paper a method is proposed to design the control law and the switching hyperplane of the sliding mode control. The robustness against matched disturbance and the system stability are discussed. Simulation results of the PMLSM servodrive are given and its show good performance for the tracking in presence of input disturbance and parameter uncertainties. For the sake of comparison purposes, simulations are carried out for the same reference trajectories with a classical proportional-integral (PI) controllers. It should be noted that, the PI-type controller is still the most popularly used for motor drives, especially among permanent magnet synchronous motor drives; this is due to its relatively simple implementation. However, PI-type control methods are not enough to accommodate the variations of external disturbance and parameter uncertainties during operation. This is obvious from the given simulated results.

\section{SYSTEM DESCRIPTION AND PROBLEM FORMULATION}

Consider a dynamical system described by

$$
\begin{aligned}
& \dot{x}(t)=A x(t)+B(u(t)+f(x, t)) \\
& y(t)=C x(t)
\end{aligned}
$$

where $x(t) \in \mathfrak{R}^{n}$ is the state vector, $u(t) \in \mathfrak{R}^{m}$ is the control input, $y(t) \in \mathfrak{R}^{p}$ is the output vector and $f(t, x) \in \mathfrak{R}^{m}$ represents the uncertainties and /or/ disturbances. Note that $f(t, x)$ is assumed unknown but bounded, i.e. $\|f(t, x)\| \leq f_{o}(t)$ where $f_{o}(t)$ represents known upper bound and $\|\cdot\|$ denotes the Euclidean norm. $A \in \mathfrak{R}^{n \times n}$, $B \in \mathfrak{R}^{n \times m}$, and $C \in \mathfrak{R}^{p \times n}$, are real constant matrices of the nominal system. Define the integral vector of tracking error as [8]

$$
\zeta(t)=\int_{0}^{t} e_{y}(t) d t=\int_{0}^{t}\left(y_{d}-y(\tau)\right) d \tau
$$

where $e_{y}(t) \in \mathfrak{R}^{p}$ is the tracking error, $y_{d}(t) \in \mathfrak{R}^{p}$ is the desired reference input and $\zeta(t) \in \mathfrak{R}^{p}$. Equivalently, (2) can take the following differential form

$$
\dot{\zeta}=y_{d}-y(t)=y_{d}-C x(t)
$$

The newly defined augmented system can be rewritten in the following form

$$
\dot{z}(t)=M z(t)+H(u(t)+f(x, t))+N y_{d}
$$

where 


$$
\begin{aligned}
& z=\left[\begin{array}{l}
x \\
\zeta
\end{array}\right] \in \mathfrak{R}^{(n+p)}, M=\left[\begin{array}{cc}
A & 0 \\
-C & 0
\end{array}\right] \in \mathfrak{R}^{(n+p) \times(n+p)}, \\
& H=\left[\begin{array}{l}
B \\
0
\end{array}\right] \in \mathfrak{R}^{(n+p) \times m}, \quad N=\left[\begin{array}{c}
0 \\
I_{p}
\end{array}\right] \in \Re^{(n+p) \times p}
\end{aligned}
$$

Therefore, the problem is to design a switched robust control that drive the plant state to the switching surface and maintain it on the surface upon interception. A Lyapunov approach is used to characterize this task. Also, switching surface that gives desired system dynamics on sliding will be designed. Moreover, simulation results will be given to clarify the effectiveness and robustness of the suggested method.

\section{SLIDING MODE CONTROL DESIGN}

Define the sliding function, $\sigma(z)$, as

$$
\sigma(z)=S z(t)
$$

where $S \in \Re^{m \times(n+p)}$ matrix and will be determined such that the sliding mode dynamics in the sliding surface

$$
S=\left\{z \in \Re^{(n+p)} \mid \sigma=S z(t)=0\right\}
$$

have $(n+p-m)$ desired eigenvalues. The choice of $S$ is often referred to as hyperplane design. This will be discussed latter. Now, the controller is designed to drive the system trajectories into sliding motion and once the system is in sliding motion, the controller should be keep the system in sliding motion despite the presence of uncertainties/and/ or disturbances. The reachability condition is chosen to make the sliding manifold attractive to the remaining state space. This reachability condition makes sure that the selected Lyapunov function [4]

$$
V=\sigma^{T} \sigma / 2
$$

satisfy that

$$
\sigma^{T} \dot{\sigma}<0
$$

So, define the control law as

$$
u=u_{E}+u_{R}
$$

where $u_{E}$ is the equivalent control that may be obtained from a conventional method of the linear system theory applied to the nominal system and $u_{R}$ is the robust control which is switching in nature. The control law will be developed to guarantee the reaching condition (8). Using (4) and (6), $u_{E}$ can be obtained as follows

$$
\dot{\sigma}(z)=S \dot{z}=S M z+S H u_{E}+S N y_{d}
$$

Therefore,

$$
u_{E}=-(S H)^{-1} S M z-(S H)^{-1} S N y_{d}
$$


Also, $u_{R}$ is assumed as follows

$$
u_{R}=-(\mu+\rho \beta)(S H)^{-1} \operatorname{sgn}(\sigma)
$$

in which $\mu>0, \beta \geq\|f\|, \rho \geq\|S H\|$, and $\operatorname{sgn}(\sigma)$ is the sign function of $\sigma$ that defined as

$$
\operatorname{sgn}(\sigma)=\left\{\begin{array}{lll}
+1, & \text { if } & \sigma>0 \\
-1, & \text { if } & \sigma<0
\end{array}\right.
$$

Consequently, the control law will be

$$
u=-(S H)^{-1} S M z-(S H)^{-1} S N y_{d}-(\mu+\rho \beta)(S H)^{-1} \operatorname{sgn}(\sigma)
$$

\section{Lemma:}

The control vector represented by (13) is satisfying the reaching condition given in (8).

\section{Proof:}

Using (4), (7) and (13), yields

$$
\begin{aligned}
& \sigma^{T} \dot{\sigma}= \sigma^{T} S \dot{z} \\
&= \sigma^{T} S\left(M z+H(u+f)+N y_{d}\right) \\
&= \sigma^{T} S\left[M z-H(S H)^{-1} S M z-H(S H)^{-1} S N y_{d}\right. \\
&\left.-H(\mu+\rho \beta)(S H)^{-1} \operatorname{sgn}(\sigma)+H f+N y_{d}\right] \\
&= \sigma^{T}[-(\mu+\rho \beta) \operatorname{sgn}(\sigma)+(S H) f] \\
&=-(\mu+\rho \beta) \sigma^{T} \operatorname{sgn}(\sigma)+\sigma^{T}(S H) f \\
&=-(\mu+\rho \beta)\left\|\sigma^{T}\right\|_{1}+\sigma^{T}(S H) f \\
& \leq-(\mu+\rho \beta)\left\|\sigma^{T}\right\|_{1}+\left\|\sigma^{T}\right\| S H\|f\| \\
&<0
\end{aligned}
$$

whereas $\left\|\sigma^{T}\right\|_{1} \geq\left\|\sigma^{T}\right\|$

Now, the sliding matrix $\mathrm{S}$ is chosen by assigning the system dynamic on sliding motion. So, in surface represented by (6), the system dynamics properties are determined by suitable choice of $(\mathrm{n}+\mathrm{p}-\mathrm{m})$ eigenvalues, say, $\lambda_{1}, \lambda_{2}, \ldots \ldots \ldots, \lambda_{n+p-m}$. The rest of $\mathrm{m}$ eigenvalues are assigned to be zeros [9]. However, in [10], these $\mathrm{m}$ eigenvalues are placed at some stable value, $\lambda_{*}$, and called the sliding margin. So, substituting from (11) into (4), then the system dynamics on sliding is described by

where

$$
\dot{z}=(M-H K) z
$$

$$
K=(S H)^{-1} S M
$$


is the state feedback gain matrix. Clearly, the matrix $\mathrm{K}$ can be found using pole placement method by choice the desired system eigenvalues. Therefore, if the eigenvalues $\lambda_{1}, \lambda_{2}, \ldots \ldots \ldots, \lambda_{n+p-m}, \lambda_{*}, \ldots \ldots \ldots, \lambda_{*}$ are suitably chosen, then the matrix $\mathrm{S}$ will be determined such that

$$
S(M-H K)=\lambda_{*} S
$$

Letting $M_{C}=M-H K$, and $Y=\left(\lambda_{*} I_{n+p}-M_{C}\right)^{T}$, then (16) reduces to

$$
Y S^{T}=0
$$

Using the generalized matrix inverse [11], the general solution of (17) for $S^{T}$ is

$$
S^{T}=\left(I_{n+p}-Y^{g} Y\right) W
$$

where $Y^{g}$ is the generalized inverse of $Y$ and $W$ is an $(\mathrm{n}+\mathrm{p}) \times \mathrm{m}$ arbitrary matrix that must be chosen to get matrix $\mathrm{S}$ such that $(\mathrm{SH})$ is invertable matrix.

\section{PMLSM SERVODRIVE MODEL AND SIMULATION}

To demonstrate the effectiveness and robustness of the proposed approach, simulation is done on the PMLSM servodrive. The model of the PMLSM servodrive is shown in Fig. 1 [3,12],

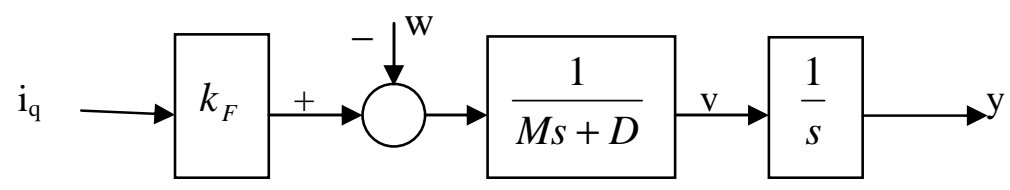

Fig. 1: PMLSM servodrive model

where $k_{F}$ is the thrust coefficient, $i_{q}$ is the command thrust current, $\mathrm{M}$ is the total mass of the moving element system, D is the viscous friction and iron loss coefficient, $\mathrm{w}$ is the external disturbance, $\mathrm{v}$ is the linear velocity and $\mathrm{y}$ is the output position. Consider the assumptions as in $[3,12]$, the nominal values of the parameters are

$$
k_{F}=20 \mathrm{~N} / \mathrm{A}, M=0.1254 \mathrm{Ns} / \mathrm{V} \text { and } D=5.2982 \mathrm{~N} / \mathrm{V}
$$

Letting $x_{1}=y$ and $x_{2}=v$, then the state space representation will take the following form

$$
\left[\begin{array}{l}
\dot{x}_{1} \\
\dot{x}_{2}
\end{array}\right]=\left[\begin{array}{cc}
0 & 1 \\
0 & \frac{-D}{M}
\end{array}\right]\left[\begin{array}{l}
x_{1} \\
x_{2}
\end{array}\right]+\left[\begin{array}{c}
0 \\
\frac{k_{F}}{M}
\end{array}\right](u+f)
$$

where $f=-w / k_{F}$. Choosing the desired eigenvalues as $-30,-35,-10$, and letting 


$$
W=\left[\begin{array}{lll}
8 & -5 & 10
\end{array}\right]
$$

then the switching matrix will be

$$
S=\left[\begin{array}{lll}
-0.5864 & -0.009 & 9.4732
\end{array}\right]
$$

Also, taking $\mu=0.2$, and using Matlab package, the simulated results of the output $\left(x_{1}\right)$, control $(u)$, robust control and $\operatorname{sgn}(\sigma)$ (sign of sigma) for a rectangular position command input (command $=4 \mathrm{~mm}$ ) are given according to the following cases:

(i) Nominal values of parameters without disturbances is presented in Fig. 2.

(ii) Uncertainty in the mass, $\mathrm{M}=2 *$ nominal, and disturbance $\mathrm{w}=20$ is applied in the shown intervals of time is shown in Fig. 3.

(iii) Uncertainty in the mass, $M=3 *$ nominal, and disturbance as in case (ii) is shown in Fig. 4.

Obviously, from the shown figures, the output position is tracking the command input in despite of the existence of disturbance and parameter uncertainties. This clarifies the robustness property of the suggested sliding mode control design approach, in comparison with the conventional PI controller, $K_{p}=3.6123$ and $K_{i}=0.9$, which is shown in Figs. 5-8.
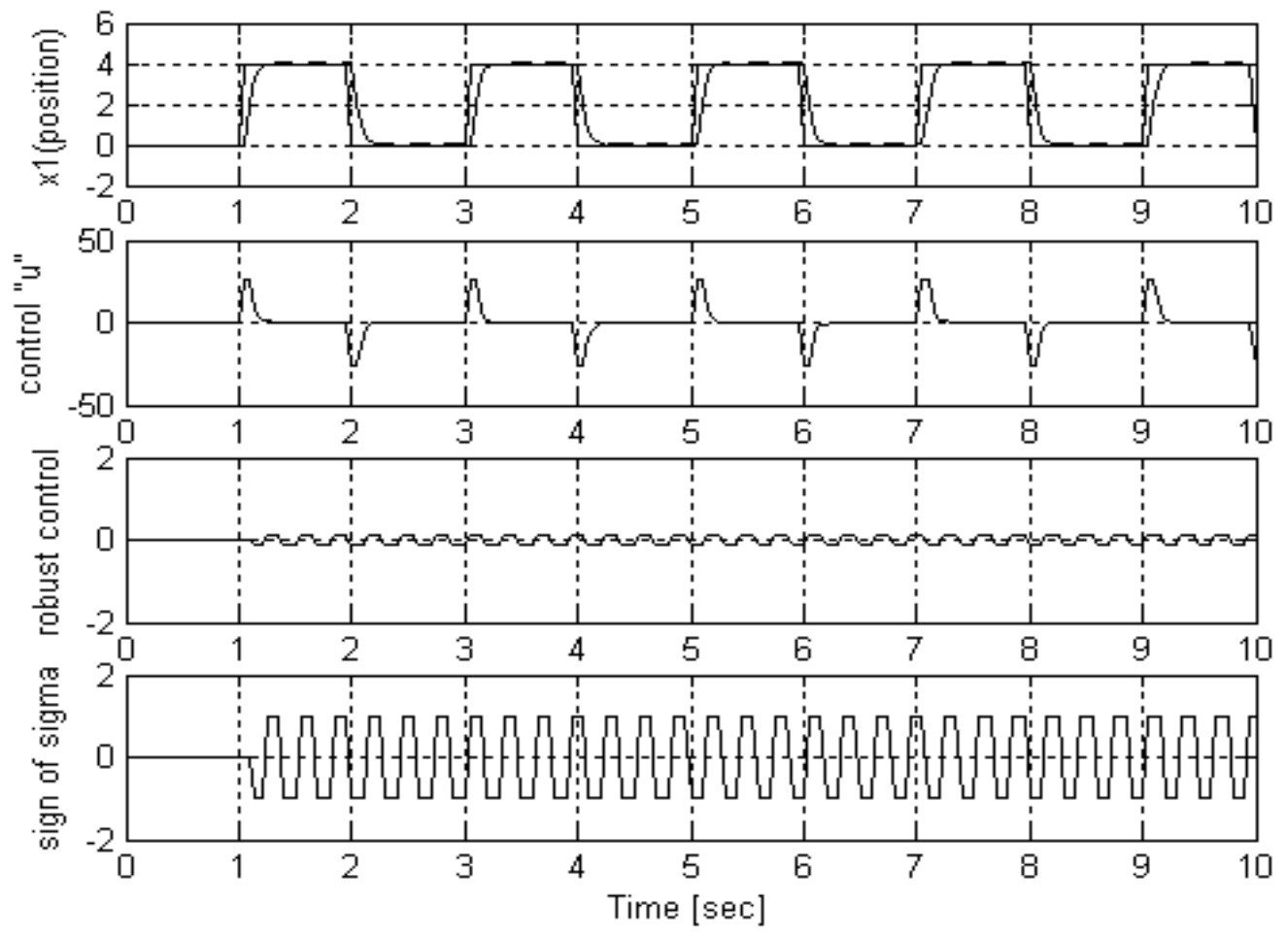

Fig. 2: simulation response of nominal system. 

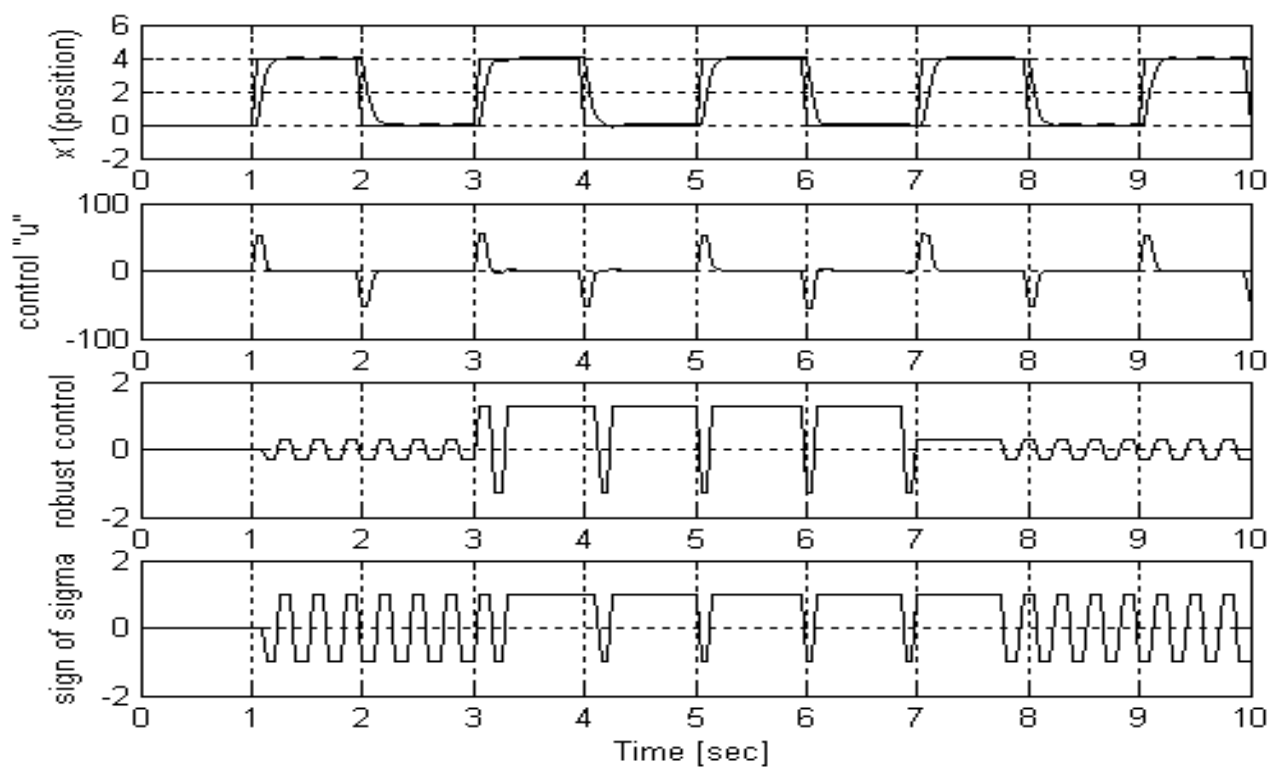

Fig. 3: simulation response with disturbance $w=20$ added from $t=3$ to $t=7$, and $\mathrm{M}=2$ nominal value.
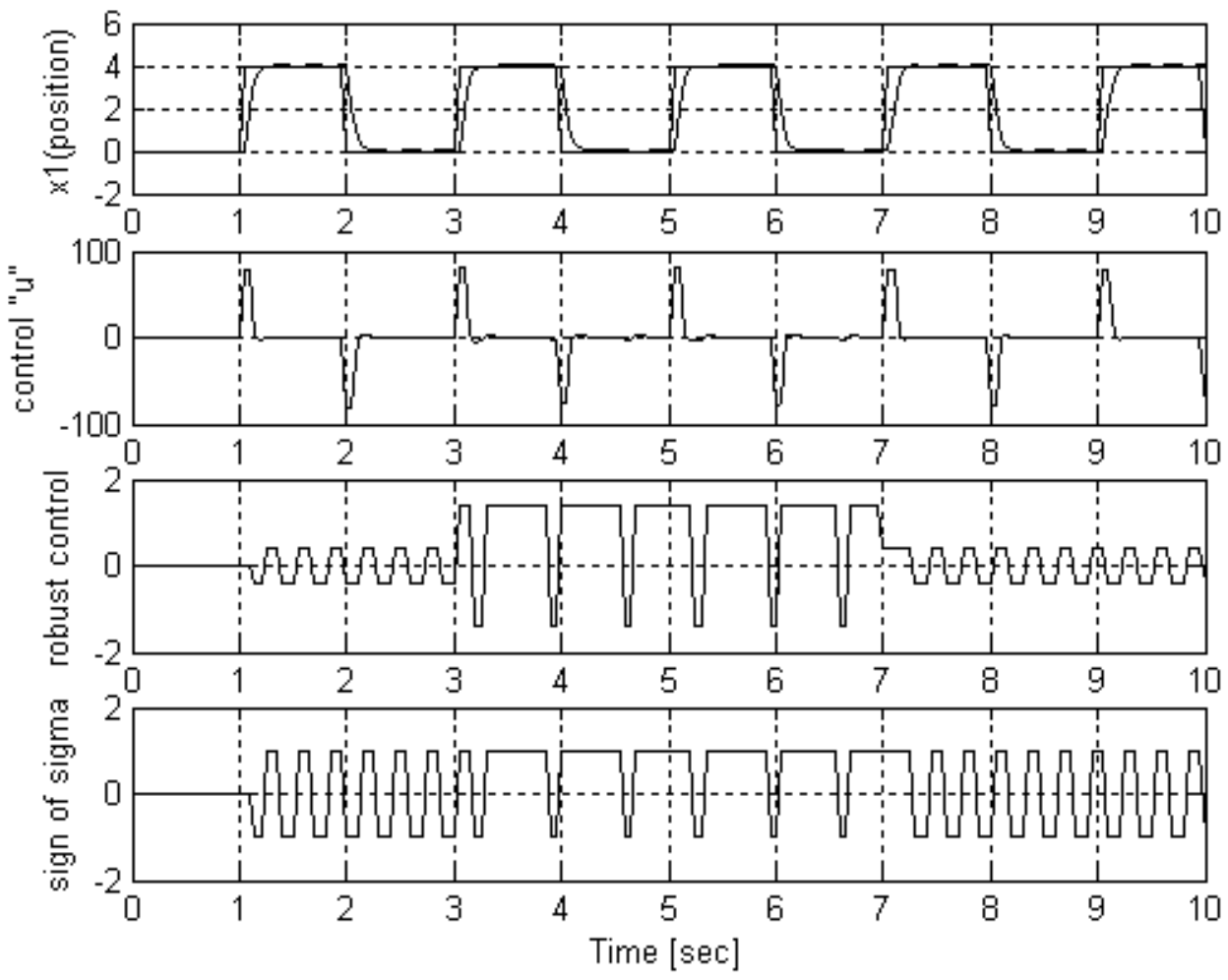

Fig. 4: simulation response with disturbance $w=20$ added from $t=3$ to $t=7$, and $\mathrm{M}=3$ nominal value 


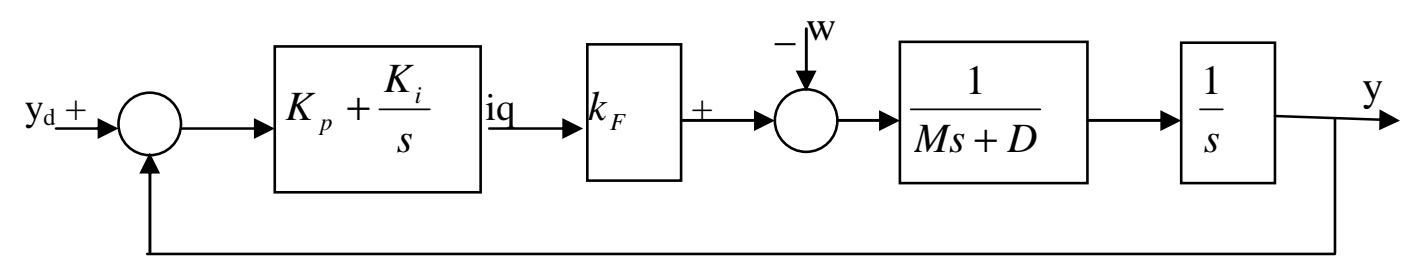

Fig. 5: PMLSM servodrive model with PI-type controller.
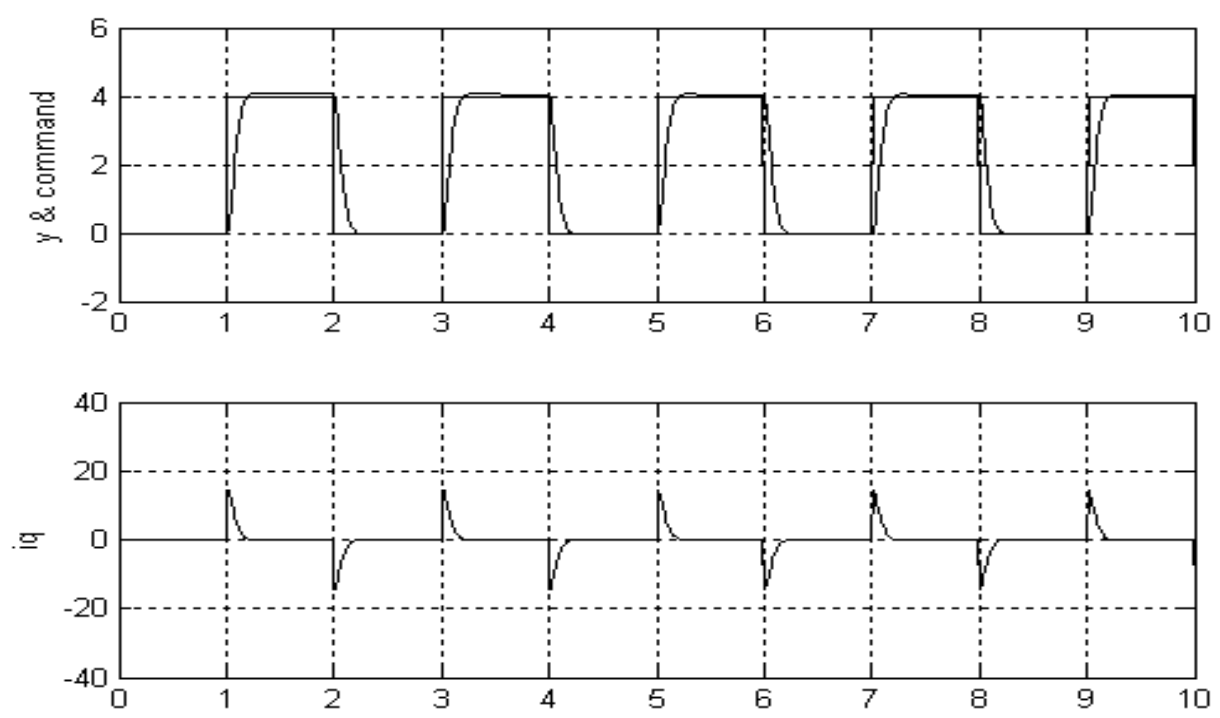

Time (sec)

Fig. 6: simulation response of nominal system with PI controller.
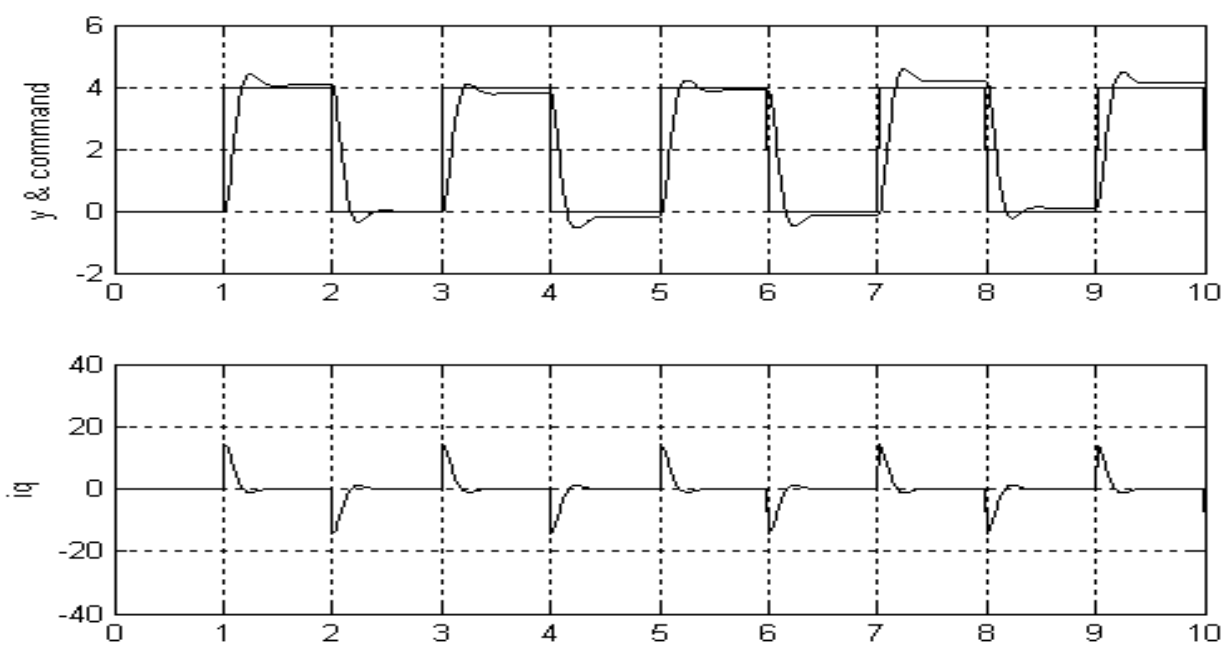

Time (sec)

Fig. 7: simulation response of system with PI controller, $w=20$ added from $\mathrm{t}=3$ to $\mathrm{t}=8$, and $\mathrm{M}=2$ nominal value. 

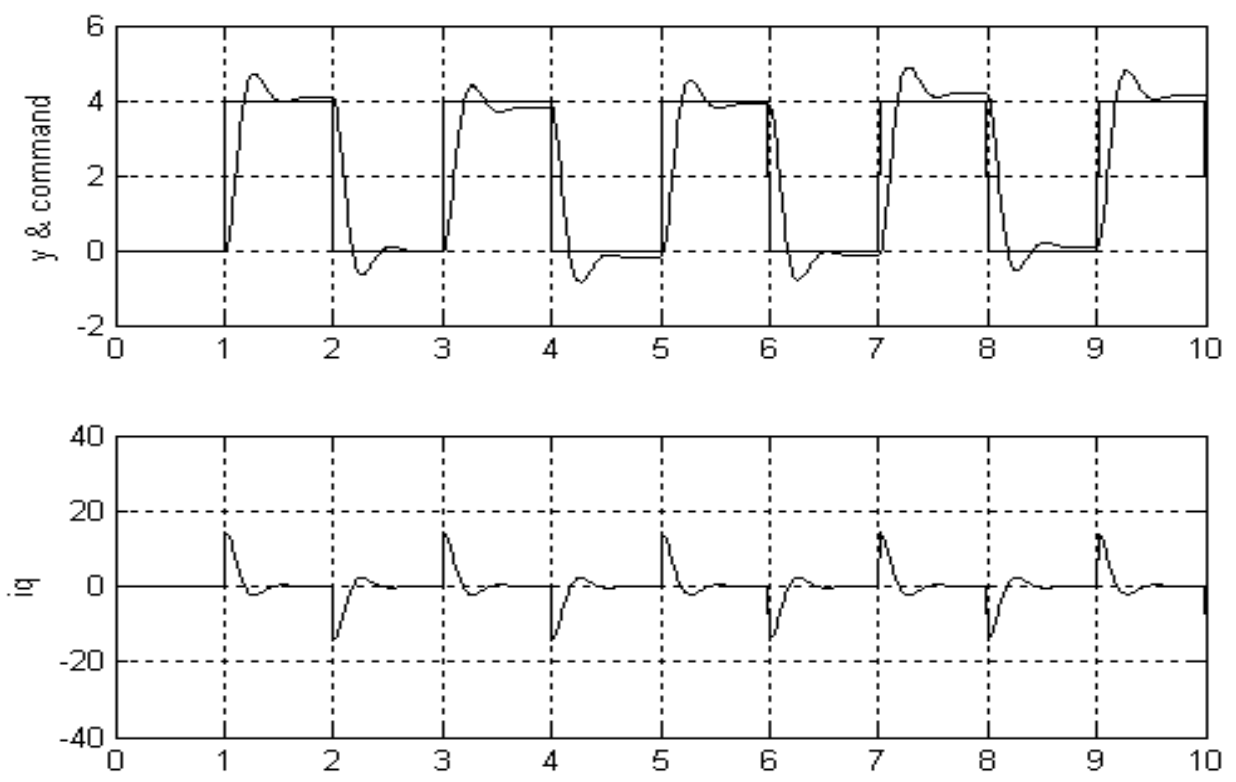

Time (sec)

Fig. 8: simulation response of system with $\mathrm{PI}$ controller, $\mathrm{w}=20$ added from $\mathrm{t}=3$ to $\mathrm{t}=8$, and $\mathrm{M}=3$ nominal value.

\section{CONCLUSIONS}

The design of a robust controller for a plant to solve the servomechanism problem has been considered. The proposed SMC control law can force the system trajectories onto the sliding motion and subsequently maintain the trajectories on this motion. Also, this control law can reject the parameter uncertainties and the external disturbance so that the output fulfills satisfactory performance. The design technique is simple and straightforward. Simulation results show the high output performance especially in presence of external disturbance and parameter uncertainties. Comparison with conventional PI-type controllers is included.

\section{REFERENCES}

[1] C.-K. Lai and K.-K. Shyu, “ A novel motor drive design for incremental motion system via sliding mode control method", IEEE Transactions on Industrial Electronics, Vol. 52, No. 2, pp. 499-507, 2005.

[2] K.D. Young, V.I. Utkin , and U. Ozguner," A control engineer's guide to sliding mode control", IEEE Transactions on Control System Technology, Vol. 7, No. 3, pp. 328-342, 1999.

[3] F.-J. Lin, C.-H. Lin and P.-K. Huang," Recurrent fuzzy neural network controller design using sliding mode control for linear synchronous motor drive", IEE Proc. Control Theory and Applications, Vol. 151, No. 4, pp. 407-416, 2004. 
[4] V.I. Utkin, "Sliding modes and their application in Variable Structure", Mir Publishers Moscow, 1978.

[5] K. Erbatur, M. O. Kaynak and A. Sabanovic," A study on robustness property of sliding mode controllers: A novel design and experimental investigations", IEEE Transactions on Industrial Electronics, Vol. 46, No.5, pp. 1012-1018 1999.

[6] J.Y. Hung, W.B. Gao, and J.C. Hung, " Variable structure control: A survey", IEEE Transactions on Industrial Electronics, Vol. 40, No. 2, pp. 2-22, 1993.

[7] J.-L. Chang, "Design of a robust controller using only output feedback to solve the servomechanism problem", IEE Proc. Control Theory and Applications, Vol. 150, No.1, pp. 28-36, 2003.

[8] K.-K. Shyu, C.-K. Lai, Y-W Tsai and D.-I Yang," A newly robust controller design for the position control of permanent magnet synchronous motor", IEEE Transactions on Industrial Electronics, Vol. 49,No. 3, pp. 558-565, 2002.

[9] J. Ackerman and V. Utkin," Sliding mode control design based on Ackerman's formula", IEEE Transactions on Automatic Control, Vol. 43, No. 2, pp. 234-237, 1998.

[10] Q.P. Ha, H. Trinh, H.T. Nguyen and H.D. Tuan, " Dynamic output feedback sliding mode control using pole placement and linear functional observers", IEEE Transactions on Industrial Electronics, Vol. 50, No. 5, pp. 1030-1037, 2003.

[11] C.R. Rao and S.K. Mitra, "Generalized Inverses of Matrices and its Applications", New York, Wiley, 1971.

[12] F.-J. Lin, C.-H. Lin and C.-M. Hong, "Robust control of linear synchronous motor servodrive using disturbance observer and recurrent neural network compensator “, IEE Proc. of Electrical Power Applications, Vol. 147, No. 4, pp. 263-272, 2000.

\section{التحكم الانزلاقى لمحرك السرفو التزامنى الخطى}

في هذه المقالة اقترحت طريقة لتصميم التحكم الانز لاقى للتحكم في نتبع الموضع للمحرك المت السرفو

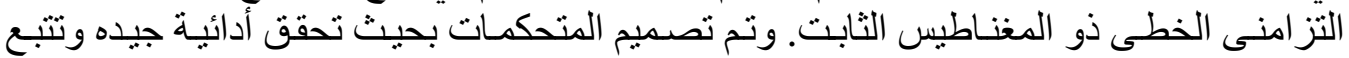

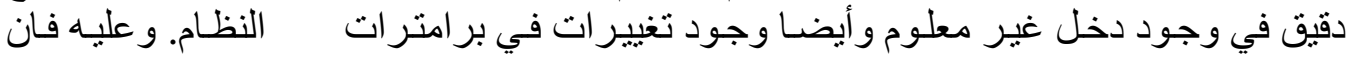

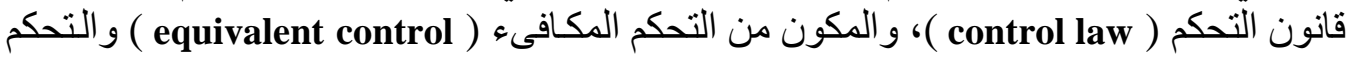

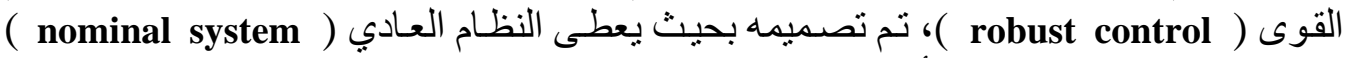

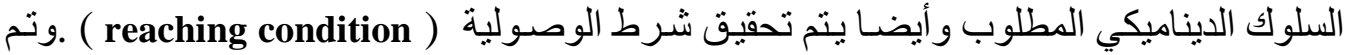

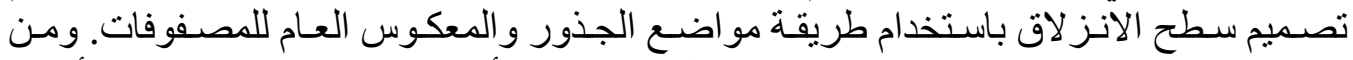

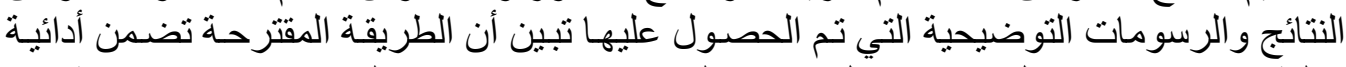

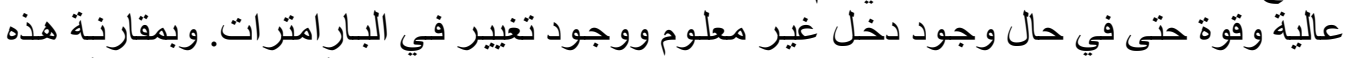

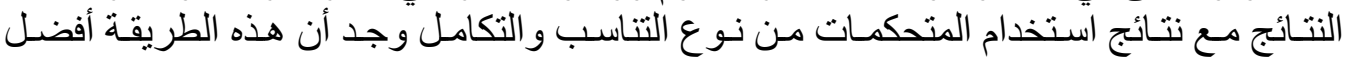

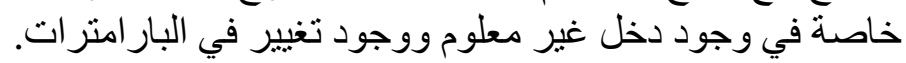

